\title{
Quantifying the resolution level where the GRACE satellites can separate Greenland's glacial mass balance from surface mass balance
}

\author{
J. A. Bonin and D. P. Chambers \\ College of Marine Science, University of South Florida, Tampa, Florida, USA \\ Correspondence to: J. A. Bonin (jbonin@ mail.usf.edu) \\ Received: 20 January 2015 - Published in The Cryosphere Discuss.: 26 February 2015 \\ Revised: 27 June 2015 - Accepted: 26 August 2015 - Published: 4 September 2015
}

\begin{abstract}
Mass change over Greenland can be caused by either changes in the glacial dynamic mass balance (DMB) or the surface mass balance (SMB). The GRACE satellite gravity mission cannot directly separate the two physical causes because it measures the sum of the entire mass column with limited spatial resolution. We demonstrate one theoretical way to indirectly separate cumulative SMB from DMB with GRACE, using a least squares inversion technique with knowledge of the location of the glaciers. However, we find that the limited $60 \times 60$ spherical harmonic representation of current GRACE data does not provide sufficient resolution to adequately accomplish the task. We determine that at a maximum degree/order of $90 \times 90$ or above, a noise-free gravity measurement could theoretically separate the SMB from DMB signals. However, current GRACE satellite errors are too large at present to separate the signals. A noise reduction of a factor of 10 at a resolution of $90 \times 90$ would provide the accuracy needed for the interannual cumulative SMB and DMB to be accurately separated.
\end{abstract}

\section{Introduction}

Mass change occurring over the ice sheets can be divided into two parts: changes due to dynamical responses of glaciers (thinning and calving) and changes due to large-scale patterns in surface melting, runoff, sublimation, and precipitation. The glacial response is known as dynamic mass balance (DMB), while the atmospherically forced signal is the surface mass balance (SMB). These two types of mass change are typically modeled or measured separately. One exception to this rule is when the ice sheet mass balance is measured by satellite gravity, such as the Gravity Recovery And Climate Experiment (GRACE); these measurements are sensitive to the sum of all mass changes, without the direct ability to separate one cause from another. In this paper, we demonstrate one theoretical way to separate cumulative SMB from DMB using GRACE, based on a priori knowledge of glacier locations on the ice sheet. Using simulations, we determine the GRACE spatial resolution needed to separate cumulative DMB and SMB around large glaciers within acceptable error limits.

In recent years, inverse least squares estimation techniques have been used to localize the smoothed signal observed by GRACE into more precise, geophysically relevant regions (Schrama and Wouters, 2011; Jacob et al., 2012; Sasgen et al., 2012; Bonin and Chambers, 2013; Luthcke et al., 2013; Wouters et al., 2013). Most often, these techniques have focused on the mass change over all of Greenland, or else within 8-16 large drainage basins covering the island. We expand this technique to include regions designed to contain the mass signal of the largest of Greenland's glaciers: Kangerdlugssuaq, Helheim, and Jakobshavn. These glacial regions experience two different physical processes atop each other: the localized DMB signal and the broader-scale SMB signal. Unlike most places in Greenland, the DMB signals in Kangerdlugssuaq, Helheim, and Jakobshavn glaciers are expected to be larger than the local SMB signal. That fact allows us to potentially separate the dynamical effects from the SMB effects in these regions, by making a pair of assumptions. First, since SMB is correlated over fairly large regions, we assume that the SMB signal across each of the large glaciers is similar to the SMB just outside the glacier. 


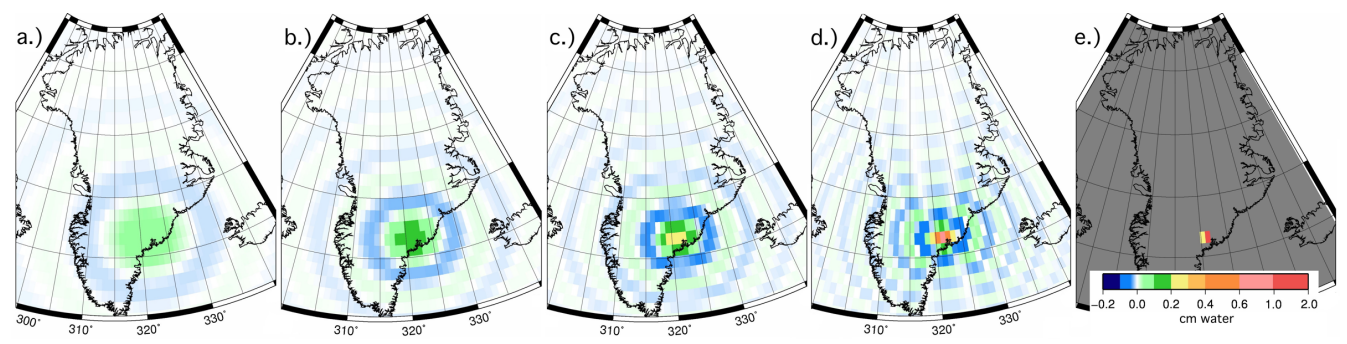

Figure 1. Impact of spatial resolution on the apparent shape and amplitude of a $1 \mathrm{~cm}$ signal over Helheim Glacier, given the a priori weight distribution in (e). Maximum degrees/orders are limited to (a) $60 \times 60$, (b) $90 \times 90$, (c) $120 \times 120$, (d) $180 \times 180$, and (e) the original $1 \times 1$ grid cells.

Second, we assume that any local signal within the glacier region which is not defined by the broader SMB signal is caused by glacial dynamics. The latter is a reasonable assumption in the case of these three glaciers, due to the relatively large size of the expected DMB signal compared to discrepancies in local SMB relative to nearby SMB. This allows us to use two overlapping basins to separate the two independent signals: first, a large SMB basin, similar to those used in previous studies; and second, a small glacial basin covering only the area just around the glacier. The smaller basin is designed to trap the localized signal, which we know to be mostly caused by the DMB, while the larger basin will trap the underlying larger-scale signal, which we know to be mostly caused by the SMB.

From a purely mathematical perspective, the least squares approach should be able to separate a localized signal (DMB) from a wider-spread signal (SMB). However, Bonin and Chambers (2013) found out via simulation that estimating mass change via an inversion modeling method, even over relatively large SMB basins, can result in trend errors of $\sim 20 \%$ of the long-term trend signal in basins losing the most mass and approaching $100 \%$ of the trend signal in more stable basins. All else equal, the smaller the area, the greater the uncertainty in the inversion results. Because of the relatively small spatial scale of even the largest glaciers, the DMB has not previously been computed using this technique.

A significant reason inversion techniques give weak results in very small areas is due to the innate limited spatial resolution of the GRACE Release-05 (RL05) data. At GRACE's typical maximum degree/order of only 60 , a strong spatially localized signal is effectively indistinguishable from a weaker, more spread-out signal. However, at higher maximum degrees, such signals become distinct (Fig. 1) - and thus, should become separable by the least squares inversion process. However, this benefit must be balanced with the cost of greater satellite errors at higher degrees. We thus aim to answer two questions. First, how high of a maximum degree/order of gravity coefficients is needed to separate the localized, large-magnitude DMB from the broaderscale, smaller-magnitude SMB? Second, what level of satellite errors is required for current or future satellite gravity missions to separate the signals with reasonable uncertainty? In this paper, we design a series of GRACE-like simulation sets with known "truth" values to test this.

\section{Description of inversion method}

Throughout this paper, we use a modified version of the least squares inversion method described mathematically in Bonin and Chambers (2013). This technique uses a set of pre-defined "basin" shapes on a $1^{\circ} \times 1^{\circ}$ grid, including all of Greenland as well as the surrounding land and ocean area. Each basin, $i$, has a pre-defined internal mass distribution assumed; using those weights, its smoothed appearance at a particular spherical harmonic resolution, $w_{i}(\phi, \lambda)$, is computed. The goal is to determine the appropriate multiplier, $a_{i}$, for each basin, such that when converted to spherical harmonics, the set of multipliers times the shape and weight of the smoothed basins results in as close a match as possible to the simulated GRACE observations.

Mathematically, this can be written as a set of models for each latitude and longitude $(\phi, \lambda)$ :

$\operatorname{model}(\phi, \lambda)=\sum_{i=1}^{\text {Nbasins }} a_{i} \cdot w_{i}(\phi, \lambda)$.

Or, this can be written in matrix form using $n$ basins:

$\operatorname{model}(\phi, \lambda)=\left[w_{1}(\phi, \lambda) w_{2}(\phi, \lambda) \ldots w_{n}(\phi, \lambda)\right]$

$\left[\begin{array}{c}a_{1} \\ a_{2} \\ \vdots \\ a_{n}\end{array}\right]=\mathbf{H}(\phi, \lambda) \mathbf{a}$.

We can then stack the matrices $\mathbf{H}(\phi, \lambda)$ for all $m$ grid cells to form an $m \times n$ matrix $\mathbf{H}$, containing all the smoothed weight information. Given the array, $\mathbf{y}$, of simulated GRACE observations, we can estimate the array of optimal amplitudes, $\mathbf{a}$, using the least squares normal equations:

$\mathbf{a}=\left(\mathbf{H}^{T} \mathbf{H}\right)^{-1} \mathbf{H}^{T} \mathbf{y}$. 


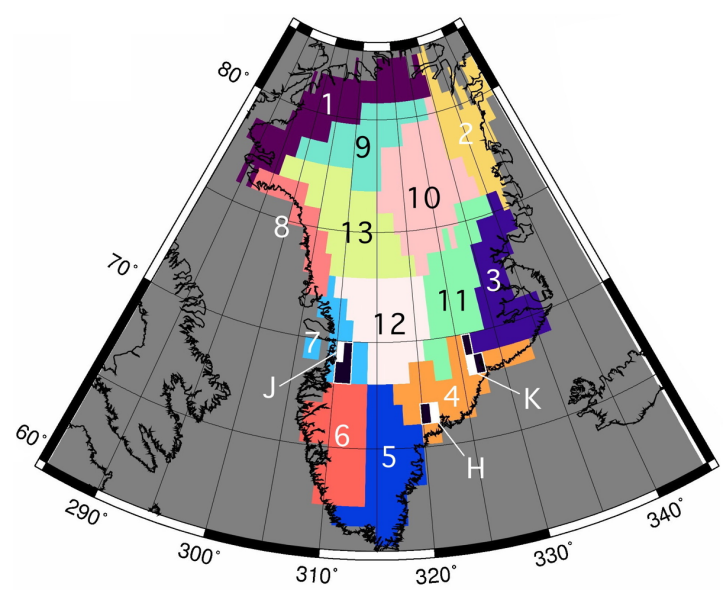

Figure 2. SMB and glacial basins for Greenland. Glacial basin (J)akobshavn overlaps with SMB basin 7, while (H)elheim and (K)angerdlugssuaq overlap basin 4 . White grid cells show the central glacier cell, while black are lesser-weight glacier cells.

We use 13 SMB basins covering Greenland (Fig. 2). These are relatively large-scale drainage basins of the area, with the coastal regions separated from the interior. To this we add 13 external basins: 4 local ocean regions and 9 nearby land regions. The latter specifically include nearby Iceland, Ellesmere Island, and Baffin Island, all of which are known to have large ice mass imbalances themselves. Unlike in Bonin and Chambers (2013), we add to this a set of three glacial basins, which overlap the SMB territory. These define three of the most significant glaciers in Greenland: Kangerdlugssuaq, Helheim, and Jakobshavn. The former two glaciers lay entirely atop SMB basin 4, while Jakobshavn is atop basin 7 . While these additional three basins do not include many other areas of active glacier dynamics, the very large signal size of these three glaciers makes them a good test case for determining if the effect of glacier dynamics could be backed out using GRACE and an inversion technique. Additional glacial basins can be added, as desired, in the future.

In Bonin and Chambers (2013), we assumed that mass was distributed evenly within each individual basin. However, that assumption was only accurate to first order, since the SMB is dominated by higher losses near the coast. Here, we instead weight the 8 external Greenland SMB basins (18), Iceland, Ellesmere Island, and Baffin Island to accentuate coastal mass change. We compute the weights using data from the RACMO2 regional climate model (Ettema et al., 2009). By summing RACMO2's SMB data from 2002 to 2012, then removing the mean at each location, we get grids of cumulative SMB anomaly, similar to GRACE's monthly mass anomalies. We use the RMS of this RACMO2 cumulative SMB data as the weights for our external Greenland basins. The internal Greenland SMB basins and other external basins are still assumed to have uniform mass distribution. The glacial basins are each dominated by a single $1^{\circ} \times 1^{\circ}$ grid cell, with $1-3$ non-zero neighboring cells whose weights are defined by modeled ice loss rates (see Fig. 4a) (Price et al., 2011). We do not assume that the actual modeled time series of glacial mass loss is correct, but merely use the model to determine the relative likely distribution of mass loss in neighboring grid cells, compared to loss within the central cell.

Although in Bonin and Chambers (2013) we determined that a diagonal constraint matrix assisted in the optimization, experimentation since has demonstrated that when using non-uniform basin weights, such "process noise" does not improve accuracy. As such, our least squares inversion technique computes the set of optimal basin multipliers using no additional constraints or regularization.

\section{Definition of the simulation sets}

Our primary goal is to quantify the accuracy of the least squares inversion method, given a fixed set of pre-defined basins and basin weights. We do this by creating multiple $1^{\circ} \times 1^{\circ}$-gridded truth simulations, converting them to (smoother) spherical harmonics, and then running them through the inversion process. The difference between the inverted basin amplitudes and the basin averages of the $1^{\circ} \times 1^{\circ}$ truth input gives the solution accuracy. We create multiple simulations to prevent coincidental similarities between the input spatial distribution and the basin weights definition from affecting the results in a way which might not occur regularly in reality.

Each simulation contains three parts: a cumulative SMB signal (Sect. 3.1), a cumulative DMB signal (Sect. 3.2), and an estimate of GRACE stripe errors (Sect. 3.3). The combination of these three pieces results in as full a simulation to the truth as we can create. By varying the SMB and DMB signals in the next two sections, we can determine the impact that misfits in the spatial distribution of the basin weights and the two ice mass signals have on the least squares results. The variation in satellite errors allows us a better statistical handle on the likely effect of the GRACE stripes. Summed together, we can determine if the combined errors are small enough to create a meaningful estimate of the truth signal - and therefore learn if this inversion technique can be used to correctly separate the SMB from DMB signals in this region.

\subsection{SMB-only simulation definition}

The "SMB-only" simulations actually include the land hydrology and oceanography signals as well as the SMB. (We call them SMB-"only" since, over Greenland, the signal is "only" SMB, not DMB or stripes.) The hydrology model used is the average of the GLDAS-Noah (Rodell et al., 2004) and WGHM (Döll et al., 2003) models. Over the oceans, we use the JPL_ECCO ocean model, run at the Jet Propulsion Laboratory (JPL) as a contribution to the Estimating the Cir- 
culation and Climate of the Ocean (ECCO), and available at http://grace.jpl.nasa.gov. It is a baroclinic model based on the MIT general circulation model (Marshall et al., 2007), forced by winds, heat flux, and freshwater fluxes from the National Center for Environmental Prediction (NCEP) operational analyses products and also assimilates satellite altimetry and other in situ observations (Fukumori, 2002; Kim et al., 2007).

To this, we add an SMB simulation. Since we had already used RACMO2 to compute the SMB basin weights, we could not directly use it to test the errors caused by misfits of those weights. So we chose to simulate plausible cumulative SMB signals using RACMO2 as a baseline. We separated the actual 2002-2012 RACMO2 signal into a long-term trend, a 12-month climatology, and the remaining residual. The long-term trend and monthly climatology together make up $83 \%$ of the RACMO2 cumulative SMB variability across Greenland, including over $95 \%$ of the coastal signal, making them the dominant terms in need of careful reproduction. The residual part contains both sub-annual variability and interannual variability, the latter of which is especially important in mass estimates over Greenland due to its connection with long-term climate change. For the SMB part of our simulations, we sought to mimic the trend, monthly climatology, and residual parts of the cumulative SMB signal by creating semi-randomized truth simulations which vary realistically but randomly from the mass distribution used in our basin weights, using the following two-part method.

\subsubsection{SMB simulation: trends and monthly climatology}

We created altered versions of the trends and monthly climatology maps, by varying the cumulative SMB signals away from the RACMO2 trends and climatology in a random but physically meaningful manner. To do so, we began by estimated typical correlative length scales, $n(\phi, \lambda)$, at each grid point from the RACMO2 cumulative SMB data. This defines the square of grid cells ( \pm no. in each direction) where the average difference from the target grid cell is at least $60 \%$ the target value itself. We computed length scales from the trend and monthly climatology maps, then averaged those 13 estimates and used that typical value for $n(\phi, \lambda)$. Length scales are generally larger among mass signals in interior Greenland than near the more highly variable coasts (Fig. 3a).

We then created generic randomized maps, $r(\phi, \lambda)$, with correlative length scales similar to those of RACMO2's trend and climatology. We first filled each map with purely random values in each $1^{\circ} \times 1^{\circ}$ land grid cell, then replaced each grid cell with the average value within its local length scale. The smoothed grid was normalized to a mean of 0 and standard deviation of 1 across all grid cells (Fig. 3b).

We then took each actual RACMO2 trend/climatology map, $x^{\mathrm{RACMO}}(\phi, \lambda)$, and perturbed it by a constant, $\alpha$, times one of the spatially correlated random maps, $r(\phi, \lambda)$ :

$x^{\operatorname{SIM}_{(1}}(\varphi, \lambda)=x^{\mathrm{RACMO} 2}(\varphi, \lambda)+\alpha \cdot r(\varphi, \lambda) \cdot\left|x^{\mathrm{RACMO} 2}(\varphi, \lambda)\right|$.

We chose $\alpha=0.5$, or a variability of $50 \%$ away from the original signal in any $1^{\circ} \times 1^{\circ}$ bin. Figure $3 \mathrm{c}$ shows an example of this technique on the trends, after subtracting off the original RACMO2 trends for visibility's sake. This technique means the high-signal coastal areas contain most of the variation, while the quieter interior of Greenland is adjusted with smaller variations away from the expected trends. The signals are not identical to RACMO2, but their differences are spatially correlated, as would be expected from physical processes such as changes in regional temperature and melting, or in precipitation. Fifty different $1^{\circ} \times 1^{\circ}$ gridded simulations of trends and climatology were created over Greenland, Iceland, and the ice-covered parts of Ellesmere and Baffin islands.

For both trends and climatology, we are probably creating a conservative estimate, since RACMO2 has been determined to have much less than $50 \%$ error (Ettema et al., 2009). However, error estimates in such studies have focused on the errors in the total mass change over all of Greenland, not the mass change in a far smaller area like a single grid cell. Since positive and negative errors will tend to average out over large areas, we presume that local $1^{\circ} \times 1^{\circ}$ RACMO2 errors will be significantly larger than global ones. Since we have no information on how much larger the local errors really are, we choose to err on the side of caution and create differences away from our basin weights larger than what we are likely to encounter in reality.

\subsubsection{SMB simulation: sub-annual and interannual variability}

While the trends and climatology describe the strongest parts of the RACMO2 cumulative SMB estimate, $17 \%$ of its variance is driven at other frequencies, including significant interannual variability. To simulate both higher- and lowerfrequency variability in the simulated data, we used a random walk process. We first created a series of the random, locally correlated maps described previously, one for each desired month of simulated data. We then used an autoregressive process such that the simulation at month $i+1, x_{i+1}^{\mathrm{SIM}}$, is a weighted combination of that month's random map, $r(\phi, \lambda)$, and the final map of the previous month, $x_{i}^{\mathrm{SIM}}$ :

$x_{i+1}^{\mathrm{SIM}}(\lambda, \phi)=\beta \cdot x_{i}^{\mathrm{SIM}}(\lambda, \phi)+[1-\beta] \cdot r(\lambda, \phi)$.

For $\beta$, we used the local autocorrelation of the RACMO2 residuals at a 1-month lag (Fig. 3d), which is typically over 0.9 . Once the entire randomized time series was created, we removed the mean and multiplied each grid cell by the standard deviation of the RACMO residual (excluding trend and climatology) in each grid cell. This gives the coastlines more 

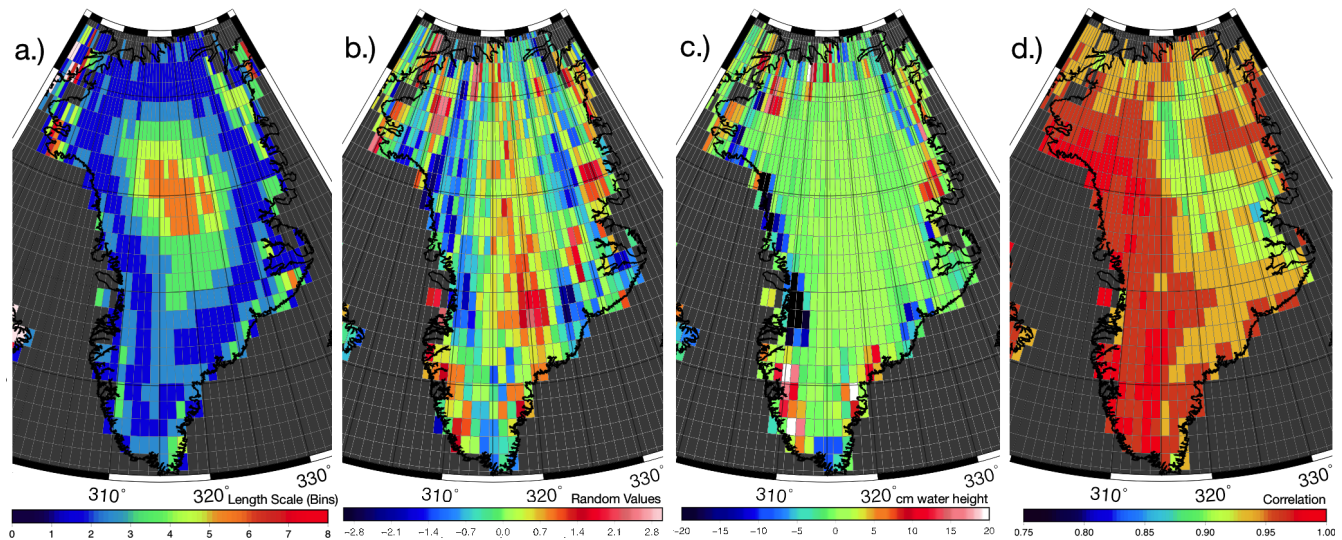

Figure 3. Example components of the simulation-building process: (a) the typical RACMO2 length scales as defined by the trend and climatology; an example of the random part of the trend signal made using this spatially correlated randomization, before (b) and after (c) applying the RMS-based amplitude weighting; and (d) the 1-month-lag autocorrelation of the RACMO2 residuals, used for the time component of the interannual and subannual simulation creation.

variability, as they have in reality, while retaining spatial correlations with the nearby grid cells and temporal correlations with neighboring months.

Each cumulative SMB simulation series is made from the summation of trend, climatology, and random-walk pieces, for each month. We created 50 simulations of 11 years of cumulative SMB simulation, designed to represent the GRACE years 2002-2012. To these, we added the modeled hydrology and oceanography series, to form the final "SMB-only" simulation truth series. We transformed these into spherical harmonic representations of maximum degree/order 60, 75, 90, 120 , and 180 for use in the least squares inversion process. The difference between the inverted results of the SMB-only simulations will estimate the sensitivity of the least squares process to imperfect SMB basin definition and weights.

\subsection{DMB-only simulation definition}

In comparison, the set of simulated cumulative DMB signal is artificially simple. We considered using a random walk process, similar to that used in the residual SMB simulation, but decided to avoid such unnecessary complexity. Firstly, we did not have access to good, monthly measurements of the mass signal in any of the three glaciers we were looking at, so we had no clear estimate of the expected variability, particularly at sub-annual frequencies. Secondly, the glacial basins are only 2-4 grid cells in size, and are each dominated by a single central grid cell, so there is minimal concern about signal overlap from nearby glacial bins with vastly different temporal signals. Instead, we kept things simple and manufactured a piecewise linear truth signal for each glacial basin (Fig. 4c). The simulated DMB signal is of roughly comparable magnitude to modeled estimates (Howat et al., 2011) and is thus much larger than the cumulative SMB signal is, though across a far smaller area. Everything outside the near- glacier regions in Fig. 4a is set to zero (since the signals there are already included in the "SMB-only" simulations).

We expect misfit errors from the cumulative DMB to arise from the imperfect basin weightings we gave to the noncentral glacier cells. To test how large an effect that has, we created an ensemble of 50 simulated cumulative DMB series, each to maximum degree/order $60,75,90,120$, and 180. Each run has the same total DMB signal per glacier, but we altered the spatial distribution of that signal slightly each time (for example, Fig. 4b vs. Fig. 4a), via the following method.

We first computed the average weight originally given to the non-central grid cells ( $\left.W_{\text {sides }}\right)$ during the definition of the glacier basins. We then altered the glacier's original grid cells (including the central cell) by adding half of $W_{\text {sides }}$ times a random value (computed with a mean of 0 and variance of 1). Those neighboring grid cells which originally had zero weights were shifted away from zero by randomized weights a tenth as large as $W_{\text {sides. }}$. The new weights around the glacier were then summed and normalized by the original sum of weights. This results in the total DMB signal strength distributed differently spatially among the 50 DMB simulations, and always non-identically to the glacier basin weights. The difference between the inverted results of the DMB-only simulations will estimate the sensitivity of the least squares process to imperfect glacier basin weights.

\subsection{Stripe-only simulation definition}

Since north-south stripe errors dominate any individual map made from unconstrained, unsmoothed GRACE data, we have created a series of simulated stripes to approximate their impact on the least squares inversion results. The stripe simulation technique is based on an observation by Swenson and Wahr (2006) that due to the north-south stripes, same-order odd-degree harmonics tend to correlate, as do same-order 

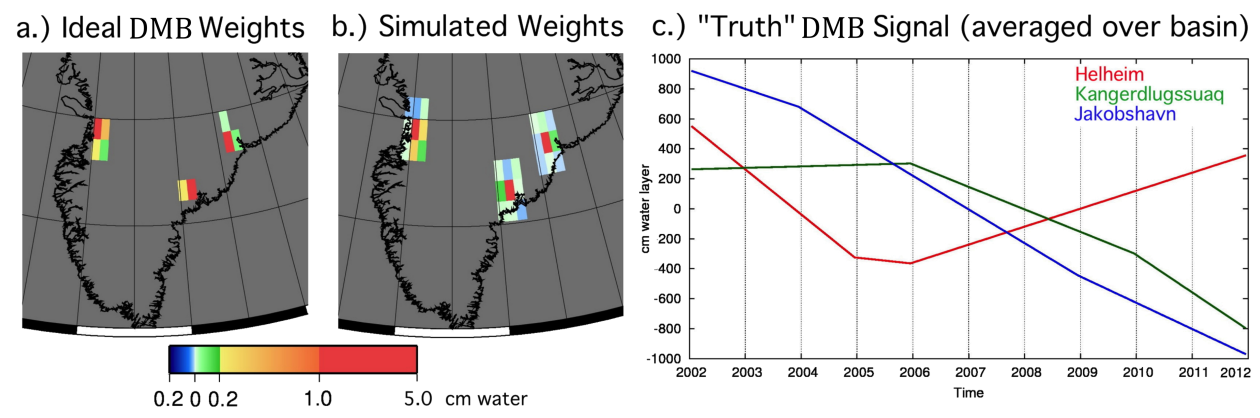

Figure 4. (a) Ideal DMB weights used as basin definitions, (b) an example of the relative weights used in the DMB truth data, and (c) the truth DMB signal for each glacier.

even-degree harmonics. Bonin and Chambers (2013) demonstrated that, given the real GRACE variances at each spherical harmonic as well as correlations with other harmonics, one can make randomized sets of simulated "GRACE-like" stripes.

We use the variances and correlations from the standard RL05 GRACE solutions from the Center for Space Research (CSR), with the AOD1B ocean dealiasing monthly averages added back. We create stripe-only simulations from harmonic cases $60 \times 60,96 \times 96$, and $120 \times 120$. The first two series are freely available on the Physical Oceanography Distributed Active Archive Center (PODAAC) website (ftp://podaac.jpl.nasa.gov/allData/grace/L2), while the latter is an experimental case run in the same manner and kindly provided by Himanshu Save at CSR. Despite the slight mismatch in maximum degree, we represent the errors for the $90 \times 90$ simulation cases with the $96 \times 96$ stripes.

To best simulate stripe errors, we remove as much of the geophysical signal as possible, to end with what we hope is mostly errors in GRACE. Thus, we removed the ocean and hydrology models used previously, as well as the RACMO2 model over Greenland and Antarctica. None of these models are perfect, so we fit a mean, trend, annual, and semiannual signal to what remained. We know that much of the remaining trend and annual signal is important geophysical signal, but some stripes also fall into those categories. To further separate that, we pulled aside only the trend/annual components of the harmonics which explained at least $50 \%$ of that harmonic's full variability. That fraction is added to the "model" and removed from the "residual". The result is a set of "model" maps that do not visibly show stripes, and a set of "residual" maps that are heavily dominated by stripes (Fig. 5a and b).

We calculated the actual variance and harmonic crosscorrelations from these residual GRACE series, then used the technique in Bonin and Chambers (2013) to make randomized sets of north-south stripes with approximately the same spatial distribution as what is actually seen in GRACE (Fig. $5 \mathrm{c}$ ). We created 50 randomized variations of the stripes for each GRACE series (degrees 60, 96, and 120). The stripe simulation technique begins to break down at high degrees/orders, overweighting the stripe amplitude within $\sim 5^{\circ}$ of the poles at maximum degree 96 and $\sim 10^{\circ}$ of the poles at maximum degree 120 . To reduce this false effect, we were forced to apply a latitude-based normalization scheme for the $96 \times 96$ and especially the $120 \times 120$ simulated stripes. This reduced the simulations' bin-based RMS to levels matching the original stripe RMS for each maximum degree.

We chose to create simulated stripes, rather than directly use the residual signal as the GRACE errors because a close look at the residuals reveals that some probably real interannual signal remains in several of the coastal Greenland basins, even after the trend and/or annual fit and removal. This is caused by an imperfect SMB/glacial model and the fact that not all remaining signal is perfectly linear or annual. In terms of the simulated stripes, it implies that our stripe estimates will tend to somewhat overstate the true north-south stripes, since the variance of the remaining interannual signal will go into simulated stripes. This makes our stripe simulation a slightly conservative estimate of the expected GRACE errors.

Numerous techniques exist to reduce these stripe errors, including a variety of spatial smoothings, correlation-based destriping methods, and spatial and temporal constraints; however each one necessarily impacts the signal along with the error. More critical to our interest here, they effectively reduce the spatial resolution of the GRACE data, by damping both error and signal at higher degrees/orders. To use any such post-processing method would undo the benefits of inverting a high-resolution series, making it more difficult to reach the needed resolution to separate SMB from DMB signals. As such, we choose to use no spatially based stripereduction method.

However, we do use one simple technique to reduce the errors at no spatial cost: applying a year-long temporal moving window to the data. This is useful since a majority of the stripe RMS occurs at periods of less than 1 year. For example, in the $120 \times 120$ case, removing the high-frequency temporal signal reduces the bin-by-bin stripe RMS to $15 \%$ or less their original size around the globe. Due to the way 


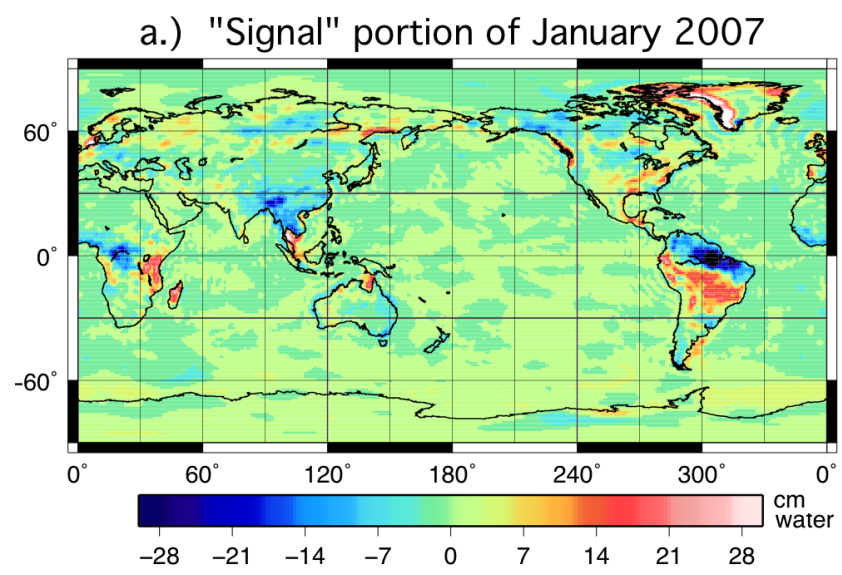

b.) "Noise" portion of January 2007

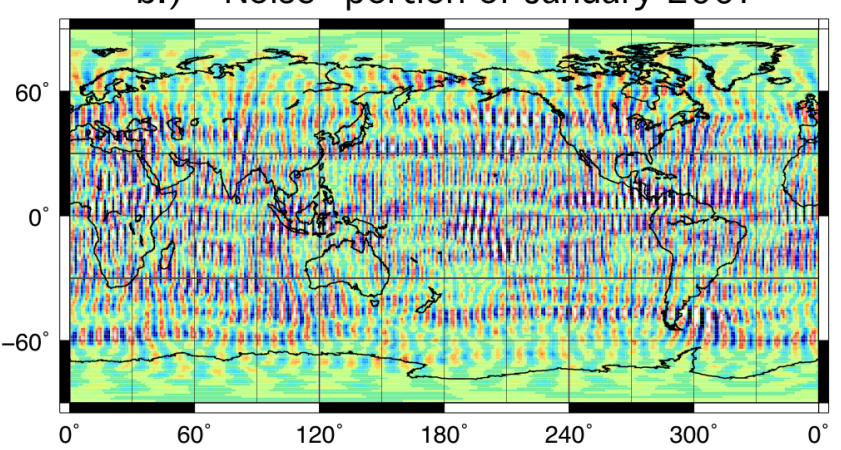

c.) Example month of randomized stripes

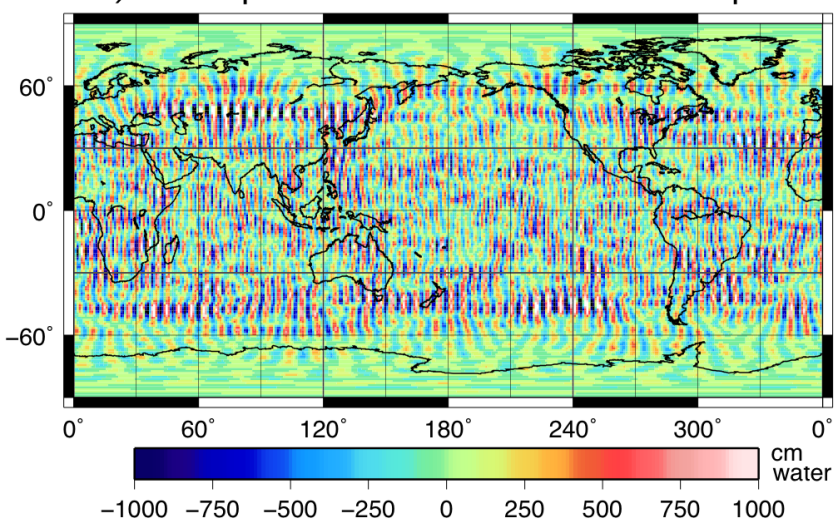

Figure 5. $96 \times 96$ (a) "model" and (b) "stripe residual" for January 2007, and (c) an example month of simulated $96 \times 96$ stripes.

basin-scale analysis averages through stripes, this results in yearly averaged stripe basin RMS values of only about onethird the size of the full stripe basin RMS. And while this will remove or dampen any high frequency truth signal, it is the longer-period Greenland ice mass signal we are most interested in for climate change, which means there is only a limited cost to removing some stripes in this way. All of the analysis in the following section uses yearly smoothed data.

\section{Analysis and discussion}

We thus have three sets of simulations, to test the three likely types of error in the least squares inversion process. The SMB-only simulation set will be used to test the impact that imperfect definitions of SMB basins and basin weights will have on the inverted results. The DMB-only simulation set will be used to test the impact that imperfect glacial basin weights will have on the inverted results. And lastly, the stripe-only simulation set will be used to test the impact that satellite errors have on the inverted results. Since our least squares inversion routine is perfectly linear, the errors of the inversion of a summed version of the three simulation pieces are the same as the sum of the inverted errors of the three individual pieces. However, by separating the simulation into three known pieces, we can determine from which part different errors arise, and thus learn which is the most limiting factor to getting accurate results using the least squares inversion technique over Greenland.

To do so, we used the least squares inversion method on each of the 50 SMB-only, 50 DMB-only, and 50 stripe-only simulation sets, for smoothed versions of maximum degrees $60,75,90,120$, and 180 each. For each inversion, we fit to the full set of SMB, glacial, and external basins. We then subtracted each simulation's estimated inverted basin amplitudes $\left(a_{i}\right)$ from the original truth simulation averaged over each basin. To create easily comparable statistics out of all this data, we compute the RMS of this difference for each simulation, then take the ensemble mean of that RMS difference for each group of 50 randomized simulations. We call this the "average basin error" in each location, for each simulation set.

In Sect. 4.1, we compare each SMB truth input to its inverted response, to determine the errors caused by using imperfect SMB basins in the least squares method. Sect. 4.2 similarly calculates the errors due to the imperfect glacier basins, and Sect. 4.3 shows a visualization of the sum of both types of basin misfit errors. In Sect. 4.4, we determine how large the satellite errors can be, when combined with the total basin misfit errors, to allow for a signal-to-noise ratio of 2. We then compute the RMS of GRACE's satellite errors, to determine if either the current GRACE or a future probable satellite gravity mission might be able to accurately separate the glacier signal from the SMB signal.

\subsection{Misfit errors due to imperfect SMB basin weights}

Figure 6 shows the average basin error from the SMB-only simulation set, for each of the 13 SMB basins and the three glacial basins, using yearly averaged data. The effect of spatial resolution is seen clearly: with increasing maximum degree/order, the errors decrease. This demonstrates the ability of the least squares inversion technique to correctly partition SMB signal, so long as the basins it is trying to fit to are sufficiently resolvable by the limited set of spherical harmonics. 

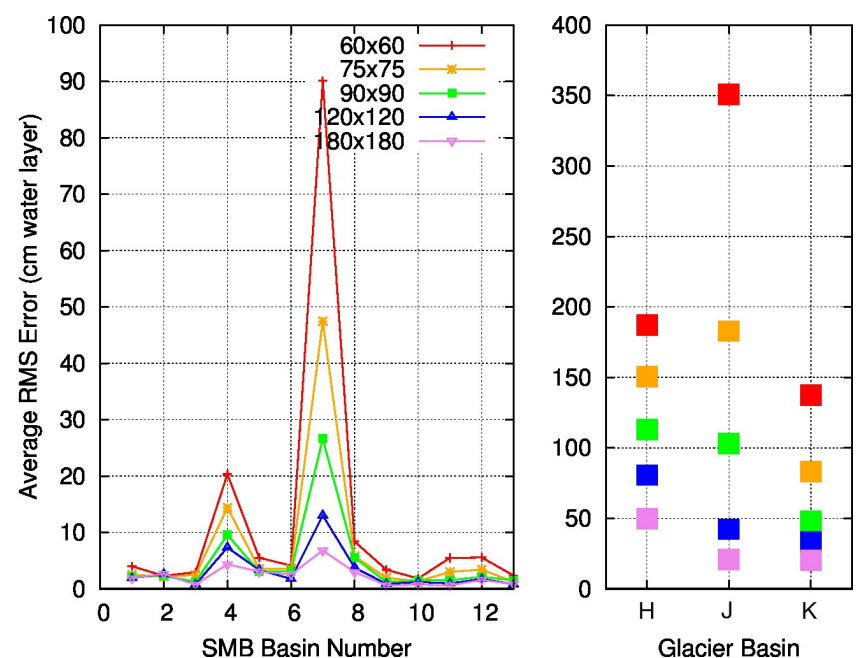

Figure 6. Average RMS difference from truth per basin for the SMB-only simulations, for data of increasing maximum degree, in SMB basins and glacial basins. Yearly windowing applied.

As the maximum degree is lowered, the biggest degradations are seen in basin 7 (which overlaps with Jakobshavn Glacier) and basin 4 (which overlaps with the other two glaciers), with particularly big changes seen as the maximum degree drops from 90 to 75 to 60 . In the case of basin 7 and Jakobshavn Glacier, the two overlapping basins have large and consistently anti-correlated error time series, particularly in the $60 \times 60$ case. At low spatial resolution, the inversion technique cannot appropriately separate the spatial maps of basins 7 and Jakobshavn, and it tends to place some of the signal that belongs in one basin into the other. Similarly, basin 4's response reflects the errors from Helheim and Kangerdlugssuaq glaciers. We hypothesize that basin 7 sees a significantly stronger signal than basin 4 because it is the smallest of the SMB basins, and because a large percentage of its high-signal coastline is also covered by the Jakobshavn basin. In comparison, the SMB signal in basin 4 is more widely distributed than the nearby glacial DMB signals, it has a significant amount of area not also covered by the glacial basins, and its two glaciers are not losing mass at identical times and rates and may counter each other's effects at times.

Even near Jakobshavn, however, the strength of this error is highly sensitive to the spatial resolution used. For example, the basin 7 and Jakobshavn SMB-misfit errors are cut in half merely by increasing the spatial resolution from $60 \times 60$ to $75 \times 75$, and to a sixth by maximum degree/order 120 . In practice, the worst of the errors caused by inexact SMB basin definitions could be avoided via an accurate higherresolution GRACE series.
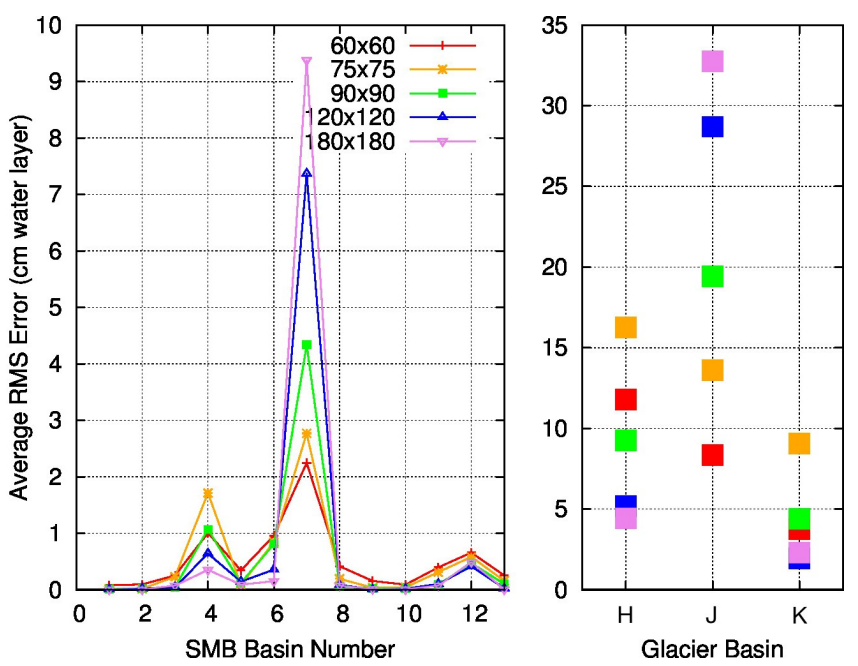

Figure 7. Average RMS difference from truth per basin for the DMB-only simulations, for data of increasing maximum degree, in SMB basins and glacial basins. Yearly windowing applied.

\subsection{Misfit errors due to imperfect glacial basin weights}

Figure 7 shows the average basin errors from the DMB-only simulation set, for each of the 13 SMB basins and the three glacial basins, using yearly averaged data. In most basins, increasing the maximum degree/order from 60 to 90 (or above) reduces the errors. However, in the critical basins 4 and 7 and the glaciers themselves, the situation is less simple. Basin 4 shows highest errors for the $75 \times 75$ cases, as do overlapping Helheim and Kangerdlugssuaq glaciers. In basin 7, the errors are inverted to what we had expected, with larger errors occurring at higher spatial resolution. There is no physical explanation for this, though we have verified that the mathematics work: the least squares results do create a smoothed pattern which closely mimics the input DMB-only signal. We view this as an example of why simulations like this one are important: just because the least squares inversion gives an answer does not mean that answer is right! In any case, the size of these glacial basin weight misfits is an order of magnitude smaller than the SMB basin misfits, making this result of secondary impact.

\subsection{Combined method errors due to imperfect SMB and glacial basin weights}

To visualize the relative size of the above misfit errors compared to the truth geophysical signals, we have plotted the inverted glacial signals from the 50 combinations of SMB and DMB simulations in Fig. 8. In the dark solid lines, we show the truth signal from each glacier basin, for comparison. The majority of the errors are driven by misfits between the SMB data and the pre-defined SMB basins, with only a small effect due to the misfit between the DMB data and the pre-defined glacial basins. 

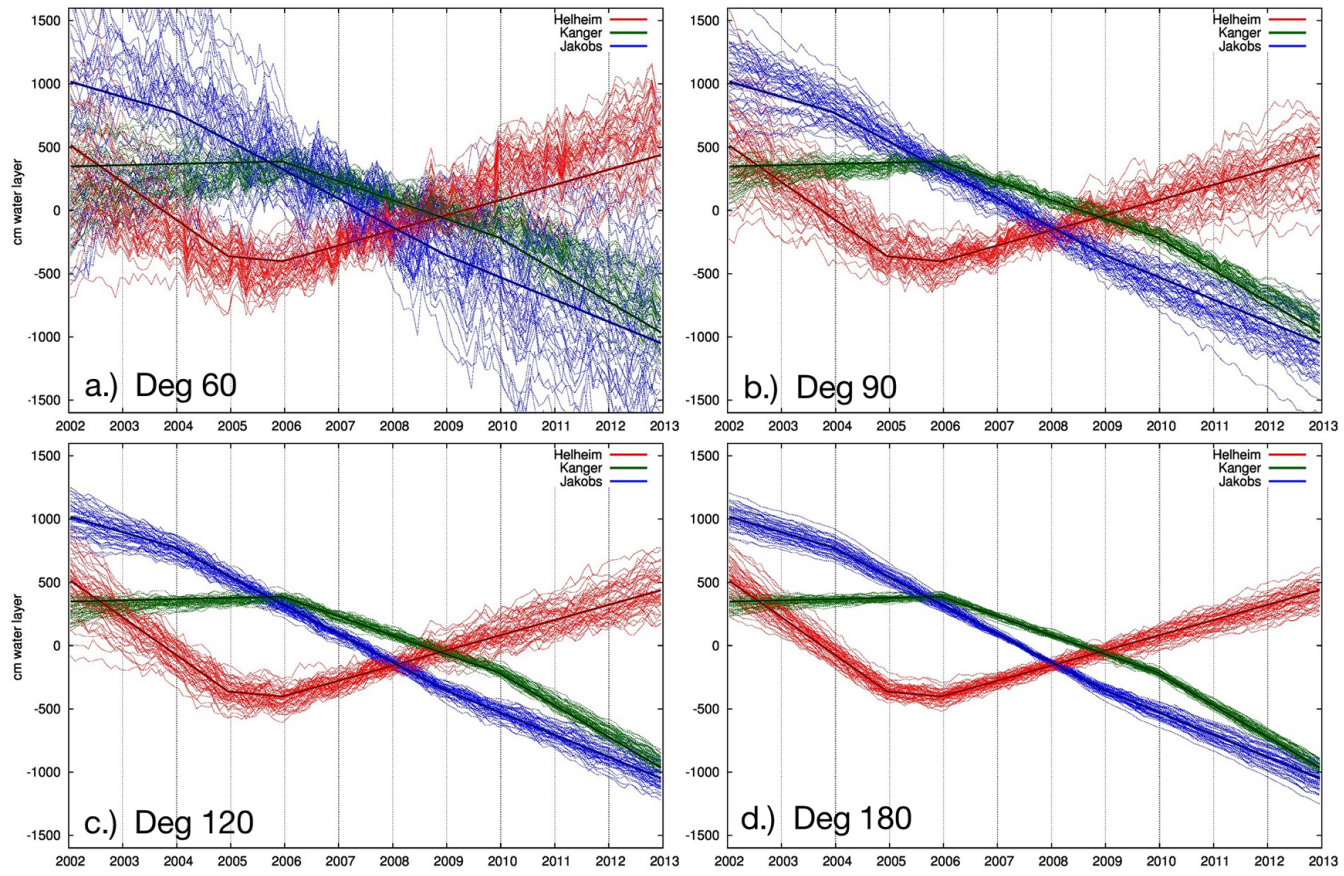

Figure 8. Visualization of the spread caused by the combined SMB and glacial basin misfit errors, at the three glaciers, for maximum degrees (a) $60 \times 60$, (b) $90 \times 90$, (c) $120 \times 120$, and (d) $180 \times 180$. Solid black lines denote the truth simulated signal per basin.
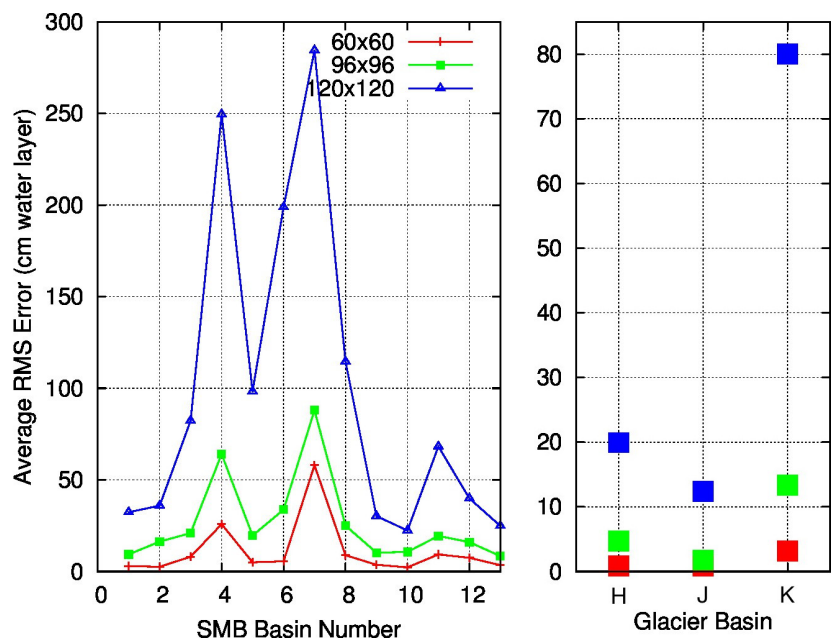

Figure 9. Average RMS error per basin for the noise-only simulations, for data of increasing maximum degree, in SMB basins and glacial basins. Yearly windowing applied.

Figure 8 demonstrates that the two types of basin weight misfit errors do not cause an insurmountable hurdle to our ability to separate the cumulative SMB from DMB signal. Error-free $60 \times 60$ solutions may not provide sufficient spatial resolution to clearly resolve interannual changes in glacial and non-glacial Greenland series, particularly in the Jakobshavn area, due to imperfections in basin definitions. However, $90 \times 90$ solutions would allow relatively small changes in inflection to be seen, if those solutions contained no satellite errors.

\subsection{Allowable vs. actual GRACE stripe errors}

Unfortunately, GRACE observations do contain satellite errors. The RMS errors caused by inverting the stripe-only simulations, after yearly smoothing, can be in Fig. 9. As expected, the errors increase as the maximum degree increases. The errors in the glacier basins are significant, and the errors in the SMB basins overlapping those glaciers grow very large, in comparison to the basin misfit errors (Figs. 6 and 7).

Figure 10a shows the SMB-misfit and glacial-basin-misfit errors from the previous sections combined in quadrature. If GRACE had no satellite errors, Fig. 10b would be the signalto-noise ratio (SNR) of the inversion technique, computed by dividing the basin RMS of the ideal truth SMB + DMB signal by these combined basin-misfit RMS errors. In this noise-free case, the SNR increases almost everywhere as spatial resolution improves. The SNR is below 1.0 (errors are larger than the signal) for most of the interior basins (9-13) at a maximum degree of 60 , and improves slightly at higher resolutions.

Most of the coastal basins have SNRs greater than 5 at all maximum degrees. However, basin 7 has the lowest SNR of the coastal basins: 0.7 at maximum degree and only 2.3 by degree 90 . Basin 4 gives nearly as poor a showing, with noise-free SNRs of 1.2 at $60 \times 60$ to 2.4 at $90 \times 90$ resolution. Even by maximum degree 180 , the SMB basins which 

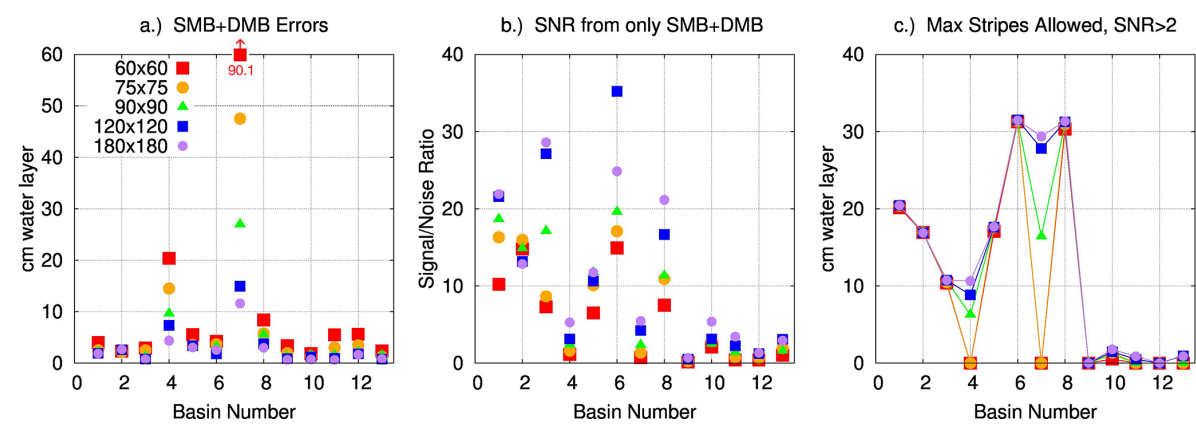

Figure 10. (a) Estimated errors caused by misfits between the SMB and DMB input data and the defined basin weights, (b) signal-to-noise ratio using only the misfit errors, and (c) the maximum stripe level allowable to result in a SNR > 2 when stripes are included. All figures use yearly windowed data.
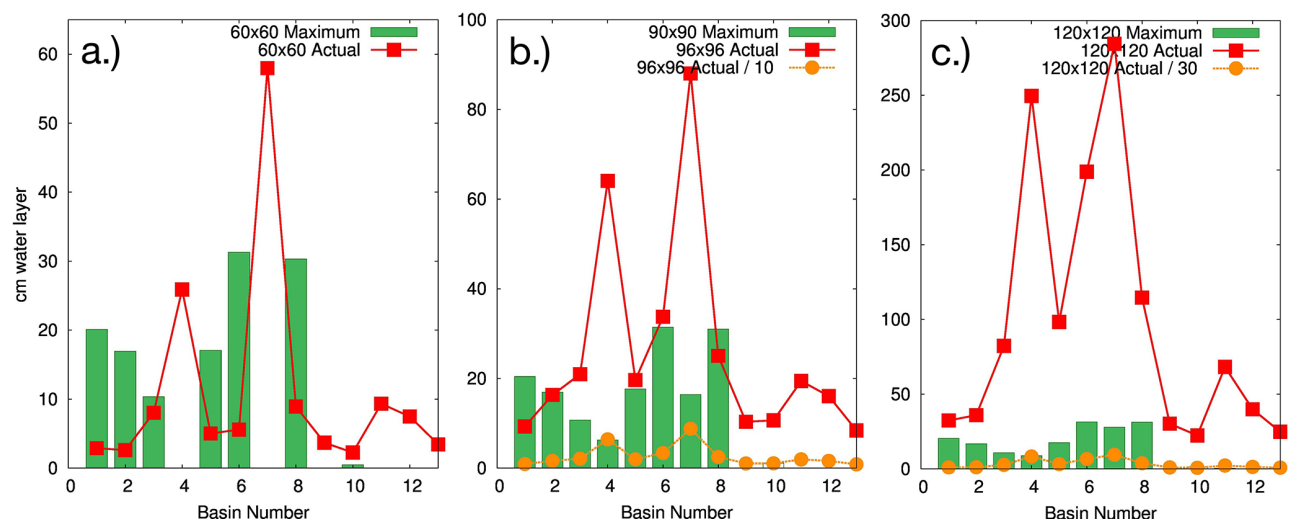

Figure 11. Comparison of maximum allowed stripes (green boxes) based on SNR >2, and the actual estimated stripes per basin (red lines) for the (a) $60 \times 60$, (b) $90 \times 90$, and (c) $120 \times 120$ cases. For (b), the actual stripe signal is from the $96 \times 96$ GRACE runs. The orange dashed lines denote the actual stripes reduced by the factors of $3.5,9$, and 30 , as needed to fall within the allowed values. All figures use yearly windowed data.

overlap the glaciers have expected noise-free SNRs of only a bit above 5 . This is concerning since the basins nearest to the glaciers are in most need of accurate separation.

Now we consider the situation if GRACE satellite stripe errors are also included. To call the cumulative SMB from DMB signals separable, we require a minimum desired stripe-inclusive SNR of 2.0 - that is, the signal RMS must be at least twice the total error RMS of the stripes and basin misfit errors combined. In Fig. 10c, we show the maximum stripe errors which meet this SNR>2 goal, given the known basin misfit errors and truth signals. We compute this using

$\mathrm{SNR}=\frac{\mathrm{RMS}_{\text {truth }}}{\sqrt{\left(\mathrm{RMS}_{\mathrm{SMB}}{ }^{2}+\mathrm{RMS}_{\mathrm{DMB}}{ }^{2}\right)+\mathrm{RMS}_{\text {stripes }}{ }^{2}}} \geq 2$,

which gives

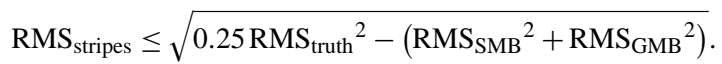

The maximum level of allowable stripes is largely independent of maximum degree/order, except in SMB basins 4 and 7. In those critical basins, it is impossible to get stripeinclusive SNRs of 2.0 at a maximum degree of 75 or below, and at $90 \times 90$ the basins remain particularly sensitive to stripe errors. As Fig. 8 previously suggested, a spatial resolution of at least $90 \times 90$ is needed to correctly separate the SMB and DMB signals into their correct basins.

Unfortunately, the actual yearly windowed inverted errors from the stripe-only simulations are large and grow larger quickly with increasing maximum degree/order (Fig. 9). Figure 11 shows a direct comparison of the possible ranges of stripe errors which allow a stripe-inclusive SNR of at least 2 (green bars), relative to the actual RMS errors found from the stripes-only simulation. The non-glacier-overlapping SMB coastal basins of the $60 \times 60$ case all have stripe errors within the acceptable SNR > 2 ranges, but by $120 \times 120$, the actual errors in all basins are much larger than needed to reach that target. In the critical glacier-overlapping basins, 4 and 7 , a $60 \times 60$ solution cannot reach an SNR of 2 , the $90 \times 90$ stripes are up to 10 times larger than the maximum allowable, and the $120 \times 120$ stripes are about 30 times too large. The trouble is two-fold: first, the GRACE stripe errors increase 
rapidly with degree, and second, the inversion technique preferentially dumps narrow signals, like stripes, into small basins, like the glacial basins, while "averaging through" more of the stripes over larger (and especially wider) basins. The allocation of stripe signal into glacier basins results in inverse signals allocated to SMB basins 4 and 7 . This counters much of the local effect and best represents the shortwavelength stripe signals, but it also badly pollutes estimates of SMB and DMB there. The negative impact of the stripes is stronger than if the inversion was done using only SMB basins and had no places specifically allocated to catch the glacial DMB signal.

\section{Conclusions}

A basin-based least squares inversion technique can theoretically be used to separate the cumulative SMB signal from the cumulative DMB signal in Greenland, assuming sufficient spatial resolution of the input data. We found that a maximum degree of $60 \times 60$ is insufficient for this task, particularly near Jakobshavn Glacier, but that a maximum degree of $90 \times 90$ can accomplish it with expected signal-tonoise ratios greater than 2 in all coastal SMB basins. Internal basins have smaller SNRs and may need to be combined into broader basins, if their far smaller mass distribution is to be correctly measured. The expected errors due to misfitting basin weights are small enough to clearly discern fairly small interannual changes in glacial signals, though we would expect weaker results for the SMB basins overlapping those glaciers. A $90 \times 90$ spatial resolution has already been achieved by today's GRACE and is plausible out of future satellite gravity missions as well.

Unfortunately, this is true in theory only. Realistically, when current GRACE noise estimates are included, a SNR $>2$ is never achievable for the SMB basins where the three targeted glaciers are located. Since GRACE errors increase far faster with degree than the inversion method's basin-misfit errors decline, this problem becomes worse as the maximum degree of GRACE increases. There is no point where the misfit errors in the inversion method (highest at low degrees) balance with the satellite errors (lowest at low degrees) to allow a good SNR. If SNR levels higher than 2 are desired, the GRACE errors would need to be brought down even further, as they depend on the inverse square of the target SNR.

Significant stripe reduction could potentially allow for cumulative SMB and DMB to be separated using the least squares inversion method, particularly if errors are also reduced via temporal smoothing, as we have done here. Taking into account yearly averaging, the GRACE noises would need to be reduced by approximately a factor of 10 at $90 \times 90$ or 30 at $120 \times 120$. This noise reduction would need to be accomplished without altering or removing the high-spatialresolution signal. We suspect that no post-processing scheme alone can currently accomplish this task, so the separation of the DMB from SMB using this method must await a new GRACE release or a future mission with smaller stripe errors.

Acknowledgements. The authors want to express great thanks to Himanshu Save at the Center for Space Research in Austin, TX, for the use of his $120 \times 120$ solutions and error estimates, as well as his helpful commentary on the research.

Support for this research was funded by the NASA GRACE Science Team program and by the NASA New (Early Career) Investigator Program in Earth Science.

Edited by: E. Larour

\section{References}

Bonin, J. and Chambers, D.: Uncertainty estimates of a GRACE inversion modelling technique over Greenland using a simulation, Geophys. J. Int., 194, 212-229, doi:10.1093/gji/ggt091, 2013.

Döll, P., Kaspar, F., and Lehner, B.: A global hydrological model for deriving water availability indicators: model tuning and validation, J. Hydrol., 270, 105-134, doi:10.1016/S00221694(02)00283-4, 2003.

Ettema, J., van den Broeke, M. R., van Meijgaard, E., van de Berg, W. J., Bamber, J. L., Box, J. E., and Bales, R. C.: Higher surface mass balance of the Greenland ice sheet revealed by highresolution climate modeling, Geophys. Res. Lett., 36, L12501, doi:10.1029/2009GL038110, 2009.

Fukumori, I.: A Partitioned Kalman Filter and Smoother, Mon. Weather Rev., 130, 1370-1383, 2002.

Howat, I. M., Ahn, Y., Joughin, I., van den Broeke, M. R., Lenaerts, J. T. M., and Smith, B.: Mass balance of Greenland's three largest outlet glaciers, 2000-2010, Geophys. Res. Lett., 38, L12501, doi:10.1029/2011GL047565, 2011.

Jacob, T., Wahr, J., Pfeffer, W. T., and Swenson, S.: Recent contributions of glaciers and ice caps to sea level rise., Nature, 482 , 514-518, doi:10.1038/nature10847, 2012.

Kim, S.-B., Lee, T., and Fukumori, I.: Mechanisms Controlling the Interannual Variation of Mixed Layer Temperature Averaged over the Niño-3 Region, J. Clim., 20, 3822-3843, doi:10.1175/JCLI4206.1, 2007.

Luthcke, S. B., Sabaka, T. J., Loomis, B. D., Arendt, A. A., McCarthy, J. J. and Camp, J.: Antarctica, Greenland and Gulf of Alaska land-ice evolution from an iterated GRACE global mascon solution, J. Glaciol., 59, 613-631, doi:10.3189/2013JoG12J147, 2013.

Marshall, J., Adcroft, A., Hill, C., Perelman, L., and Heisey, C.: A finite-volume, incompressible Navier Stokes model for studies of the ocean on parallel computers, J. Geophys. Res., 102, 57535766, 2007.

Price, S. F., Payne, A. J., Howat, I. M., and Smith, B. E.: Committed sea-level rise for the next century from Greenland ice sheet dynamics during the past decade., P. Natl. Acad. Sci. USA, 108, 8978-8983, doi:10.1073/pnas.1017313108, 2011.

Rodell, M., Houser, P. R., Jambor, U., Gottschalck, J., Mitchell, K., Meng, C.-J., Arsenault, K., Cosgrove, B., Radakovich, J., 
Bosilovich, M., Entin*, J. K., Walker, J. P., Lohmann, D., and Toll, D.: The Global Land Data Assimilation System, Bull. Am. Meteorol. Soc., 85, 381-394, doi:10.1175/BAMS-85-3-381, 2004.

Sasgen, I., van den Broeke, M., Bamber, J. L., Rignot, E., Sørensen, L. S., Wouters, B., Martinec, Z., Velicogna, I., and Simonsen, S. B.: Timing and origin of recent regional ice-mass loss in Greenland, Earth Planet. Sci. Lett., 333-334, 293-303, doi:10.1016/j.epsl.2012.03.033, 2012.
Schrama, E. J. O. and Wouters, B.: Revisiting Greenland ice sheet mass loss observed by GRACE, J. Geophys. Res., 116, B02407, doi:10.1029/2009JB006847, 2011.

Swenson, S. and Wahr, J.: Post-processing removal of correlated errors in GRACE data, Geophys. Res. Lett., 33, L08402, doi:10.1029/2005GL025285, 2006.

Wouters, B., Bamber, J. L., van den Broeke, M. R., Lenaerts, J. T. M., and Sasgen, I.: Limits in detecting acceleration of ice sheet mass loss due to climate variability, Nat. Geosci., 6, 613-616, doi:10.1038/ngeo1874, 2013. 